\title{
Aedes albopictus, VecteUR des VIRUS DU CHIKUNGUNYA et de LA DeNGUe À LA RÉUNION : BIOLOGIE ET CONTRÔLE
}

\author{
DELATTE H.*, PAUPY C.*, DEHECQ J.S.**, THIRIA J.**, FAILLOUX A.B.*** \& FONTENILLE D.**
}

Summary: AEDES ALBOPICTUS, VECTOR OF CHIKUNGUNYA AND DENGUE VIRUSES IN REUNION ISLAND: BIOLOGY AND CONTROL

Chikungunya virus (CHIKV) and dengue virus (DENV) are mosquitoborne viruses transmitted by the Aedes genus. Dengue is

considered as the most important arbovirus disease throughout the World. Chikungunya, known from epidemics in continental Africa and Asia, has up to now been poorly studied. It has been recently responsible for the severe 2004-2007 epidemic reported in the Indian Ocean (IO), which has caused several serious health and economic problems. This unprecedented epidemic of the 10 has shown severe health troubles with morbidity and death associated, which had never been observed before. The two major vectors of those arboviruses in the 10 area are Aedes aegypti and Aedes albopictus. The latest is considered as the main vector in most of the islands of the area, especially in Reunion Island. Ae. albopictus showed strong ecological plasticity. Small disposable containers were the principal urban breeding sites, and preferred natural developmental sites were bamboo stumps and rock holes in periurban and gully areas. The virus has been isolated from field collected Ae. albopictus females, and in two out of 500 pools of larvae, demonstrating vertical transmission. Experimental works showed that both Ae. albopictus and Ae. aegypti from west 10 islands are efficient vectors of dengue and chikungunya viruses. Since 2006 and all along the epidemic of CHIKV, measures for the control of larvae (temephos then Bacillus thuringiensis) and adults (fenitrothion, then deltamethrine) of Ae. albopictus where applied along with individual and collective actions (by the use of repellents, and removal of breeding sites around houses) in Reunion Island. In order to prevent such epidemics, a preventive plan for arboviruses upsurge is ongoing processed. This plan would allow a quicker response to the threat and adapt it according to the virus and its specific vector.

\section{Résumé :}

Les virus du chikungunya (CHIKV) et de la dengue (DENV) sont transmis par des moustiques du genre Aedes. La dengue est considérée comme l'arbovirose la plus importante dans le monde. Le chikungunya, connu d'Afrique continentale et d'Asie, et jusqu'à présent peu étudié, a été de 2004 à 2007 à l'origine de graves épidémies dans l'Océan Indien (OI), en Inde et en Afrique centrale, générant d'importants problèmes de santé publique et économiques. La récente épidémie dans le sud-ovest de l'Océan Indien (SOOI) a mis en exergue le caractère explosif de son émergence ainsi que la morbidité et la mortalité qui lui ont été associées, jusque-là sans précédent. Les deux vecteurs majeurs impliqués dans la transmission de ces arbovirus dans la zone Ol sont Aedes aegypti et Aedes albopictus, ce dernier étant considéré comme le vecteur principal dans la majorité des îles de la zone. Dans l'île de la Réunion, où Ae. albopictus est le vecteur du CHIKV, la lutte anti-larvaire (téméphos puis Bacillus

thuringiensis), anti-adulte (fénitrothion, puis deltaméthrine), ainsi qu'une protection individuelle et communautaire /utilisation de répulsifs, destruction des gîtes larvaires autour des habitations) ont été réalisées depuis 2006 et tout au long de la crise sanitaire. Afin de prévenir de telles épidémies, un plan de prévention des arboviroses est en cours de réalisation. Il devra permettre d'intervenir plus rapidement dès la réception des premiers signaux d'alerte, et d'adapter la réponse en fonction du virus et de son vecteur.

MOTS CLÉS : Aedes albopictus, chikungunya, dengue, la Réunion, lutte antivectorielle.

KEY WORDS : Aedes albopictus, chikungunya, dengue, Reunion Island, vector control.

tié de la population mondiale (Monath, 1994). Des épidémies de dengue ont notamment été observées dans la zone de l'Océan Indien (OI) (Zeller, 1998). Le chikungunya qui était jusqu'à récemment considéré comme une arbovirose mineure sévissant principalement en Afrique continentale et en Asie a récemment connu un regain d'intérêt suite à l'émergence du virus dans de nouvelles aires géographiques. Depuis 2004, le virus a notamment émergé dans l'OI sous la forme de graves épidémies à l'origine d'importants problèmes de santé publique et économiques. Suite à cette épidémie, le virus s'est signalé en Inde et en Afrique centrale, puis a récemment touché le nord de l'Italie, en juillet 2007 , avec plus de 250 cas signalés début septembre 2007, démontrant que l'Europe n'est pas à l'abri de ce type 


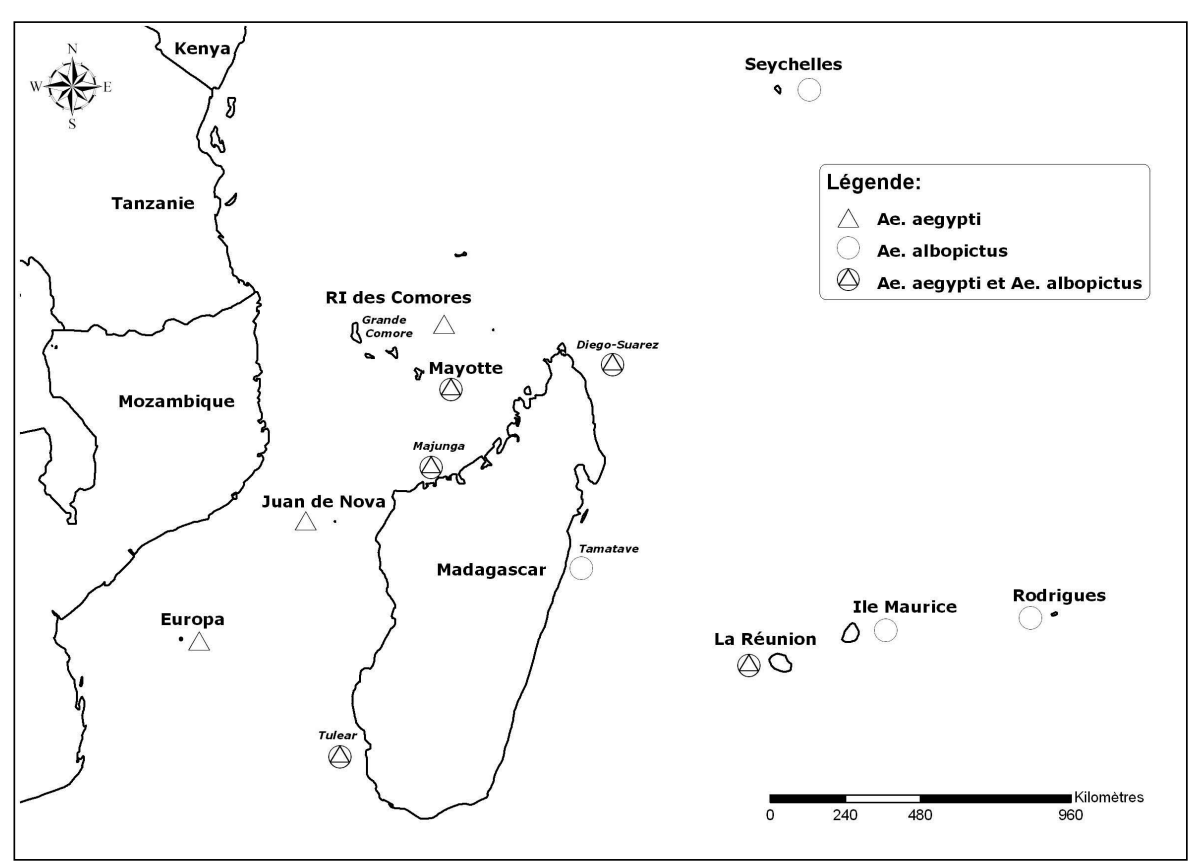

Fig. 1. - Répartition d'Aedes albopictus et d'Aedes aegypti dans la zone du sud-ouest de l'Océan Indien. d'épidémie depuis qu'Aedes albopictus, vecteur d'arbovirus, s'y est implanté (Fontenille et al., 2007).

L'émergence récente dans le sud-ouest de l'Océan Indien (SOOI) a mis en exergue le caractère explosif de l'épidémie ainsi que la morbidité et la mortalité qui lui ont été associées, jusque-là sans précédent. La zone du SOOI a été plongée dans une crise sanitaire aux répercussions socio-économiques majeures d'autant qu'aucun vaccin ni traitement étiologique spécifique n'était disponible.

Les virus du chikungunya (CHIKV) et de la dengue (DENV) sont des arbovirus dont la transmission est assurée par des moustiques du genre Aedes, principalement $A e$. aegypti. Cependant, une autre espèce, $A e$. albopictus, peut s'avérer un redoutable vecteur (Reiter et al., 2006). Dans la majorité des îles de la zone OI, cette dernière espèce est d'ailleurs considérée comme le vecteur principal aux côtés d'Ae. aegypti. Aedes albopictus est l'espèce dominante à la Réunion, aux Seychelles, à l'île Maurice, à l'île Rodrigues et dans l'est de Madagascar (figure 1).

\section{ÉPIDÉMIOLOGIE DE LA DENGUE}

L es virus de la dengue, constitués de quatre sérotypes (DEN-1, -2, -3 et -4), appartiennent à la famille des Flaviviridae et au genre Flavivirus. En termes de morbidité et de mortalité, la dengue est considérée comme la plus importante des arboviroses transmises par moustiques (Gubler, 1998). Près de 50 millions de cas et 25000 morts sont rapportés chaque année (Gibbons \& Vaughn, 2002). De nombreuses épidémies d'infections virales évoquant la dengue ont été décrites au cours des siècles en différents points du globe. Cependant, il faut attendre les travaux de Hotta et Kimura, 1943 (Hotta \& Kimura, 1952) et ceux de Sabin en 1944 (Sabin, 1952), pour que le virus de la dengue (DEN-1) soit isolé et la maladie diagnostiquée avec plus de certitude. Ces mêmes auteurs isolèrent d'un patient de Nouvelle-Guinée, un second variant viral le sérotype 2 (DEN-2) antigéniquement distinct du sérotype 1. Les deux autres sérotypes, DEN-3 et DEN4 allaient être isolés plus tard, en 1956, aux Philippines (Hammon et al., 1960). La question de l'origine géographique des virus de la dengue n'a pas encore été parfaitement élucidée, certains auteurs situant leur berceau en Afrique, d'autres en Asie. Cependant, les données épidémiologiques et moléculaires semblent conforter la thèse d'une origine Asiatique (Holmes \& Twiddy, 2003).

Sur le plan clinique, la dengue peut revêtir des formes inapparentes, des formes bénignes d'évolution non spécifique (fièvre, maux de tête, douleurs articulaires, nausées, vomissements, rash cutané), et des formes graves incluant la dengue hémorragique avec ou sans syndrome de choc et, plus rarement, des syndromes hépatiques et encéphalitiques (Rodhain, 2001).

Les îles de l'OI semblent, d'après certains récits, avoir été frappées à plusieurs reprises par des épidémies de dengue. Avant les années 1970, plusieurs auteurs rapportaient la présence de la dengue dans cette région du monde. Maurice, en 1872, et la Réunion, en 1873, auraient connu des épidémies de ce qu'on dénommait alors "fièvre rouge" (Cotholendy, 1873; Pougnet, 1890; Maccarthy \& Brent, 1946). Plus tard, en 1943, une épidémie de dengue aurait sévi aux Comores (Maccarthy \& Brent, 1946). Ce n'est qu'à partir des années 1970, 
que la dengue fut réellement confirmée en Afrique orientale et dans les îles de l'OI. Suite à ces premières observations, d'autres épidémies ont été rapportées dans la sous région, aux Seychelles entre 1976 et 1979 (Coulanges et al., 1979 ; Metselaar et al., 1980) et dans l'archipel des Comores en 1984 et 1993; affections dues aux sérotypes DEN-1 et DEN-2, respectivement (Boisier et al., 1994). Les différents sérotypes DEN-1 à 4 ont été signalés aux Seychelles avec une estimation de 38 à $50 \%$ de séroprévalence dans la population en 1995 (Yersin et al., 1999).

La Réunion a également subi la vague épidémique de DEN-2 en 1977-1978 (Mora, 1979) affectant près de 30 à $35 \%$ de la population, soit environ 170000 personnes (Coulanges et al., 1979). Cette épidémie s'est par la suite étendue à l'île Maurice (Julvez et al., 1998). En 2004, une nouvelle épidémie de dengue (DEN-1) était signalée à la Réunion (Pierre et al., 1998), affectant 228 personnes. Depuis cette date, des cas sporadiques sont régulièrement enregistrés (0 à 5 cas mensuels) sur l'île.

\section{ÉPIDÉMIOLOGIE DU CHIKUNGUNYA}

L e CHIKV, un Alphavirus appartenant à la famille des Togaviridae, a été isolé pour la première fois Tanzanie en 1953 (Ross, 1956). Cet arbovirus originaire d'Afrique se maintient au sein d'un cycle forestier faisant intervenir des primates et des moustiques Aedes selvatiques du sous-genre Aedes Stegomyia et Ae. Diceromyia (Diallo et al., 1999). Entre 1960 et 1980, le virus s'est manifesté au cours d'épidémies survenues en différents points d'Afrique tropicale : Afrique de l'Est (Tanzanie, Ouganda), Afrique Australe (Zimbabwe, Afrique du Sud), Afrique de l'Ouest (Sénégal, Nigeria) et Afrique Centrale (République de Centre Afrique et République Démocratique du Congo) où il semble circuler selon un mode enzootique (Robinson, 1955; Moore et al., 1974; Kalunda et al., 1985; Thonnon et al., 1999). Plus récemment, une épidémie touchant près de 50000 personnes est survenue en République Démocratique du Congo, à Kinshasa, en 1999-2000 (Pastorino et al., 2004), au Cameroun (Peyrefitte et al., 2006) et au Gabon (Leroy, 2007) en 2007.

En dehors de la zone afro-tropicale, le CHIKV sévit également en Asie où il a été signalé pour la première fois en 1958 en Thailande (Rao, 1966; Halstead et al., 1969). Par la suite, de nouvelles épidémies ont été décrites dans ce pays ainsi qu'aux Cambodge, Vietnam, Laos, Myanmar, Philippines, Inde et Indonésie (Jupp \& McIntosh, 1988; Johnston \& Peters, 1996). Après 20 ans d'absence, le virus est réapparu à Java, en Indonésie, en 20012003 (Laras et al., 2005) et très récemment en Malaisie. En Asie, contrairement à l'Afrique où le CHIKV peut circuler selon un mode enzootique, la transmission semble strictement inter humaine, assurée principalement par Ae. aegypti, moustique hautement anthropophile et parfaitement adapté à l'environnement urbain. Ce dernier est mieux connu pour son rôle dans la transmission de deux autres arboviroses majeures, la dengue et de la fièvre jaune. Des données issues du terrain et du laboratoire font cependant état du rôle potentiel d'un autre moustique, Ae. albopictus, dans la transmission du CHIKV en Asie (Mangiafico, 1971; Laras et al., 2005). Le virus a circulé pour la première fois en Europe, en Émilie Romagne, Italie, en été 2007, touchant plus de 250 personnes. Le vecteur était Ae. albopictus.

À la fin de l'année 2004, le CHIKV a émergé dans plusieurs îles du SOOI, tout d'abord aux Comores (Sergon et al., 2007), puis en 2005-2006 dans les îles de la Réunion, Mayotte, Maurice, Seychelles, Madagascar ainsi qu'en Inde (Pialoux et al., 2006; Schuffenecker et al., 2006; Arankalle et al. 2007). Des enquêtes sérologiques ont montré que le taux d'attaque avait atteint $38 \%$ (soit 300000 personnes) à la Réunion fin 2006 (Perrau et al., 2006), et à Mayotte (Enquête Cire de la Réunion et de Mayotte, INVS, janvier 2007) et $63 \%$ (soit 215000 personnes) en Grande Comores (Sergon et al., 2007). La circulation du CHIKV parmi les îles de la zone SOOI ne semble cependant pas nouvelle puisque la présence du virus avait déjà été notifiée aux Seychelles en 1979 (Calisher et al., 1981). Outre l'ampleur sans précédent des récentes épidémies dans la zone du SOOI, des formes cliniques sévères (méningo-encéphalites, encéphalopathies ou hépatites fulminantes), ainsi qu'une mortalité associée, ont pour la première fois été décrites pour cette maladie considérée jusqu'alors comme bénigne (Pialoux et al., 2006).

\section{LES VECTEURS}

Tusqu'à récemment Ae. aegypti était considéré comme le vecteur épidémique du virus CHIKV. Néanmoins, depuis les épidémies du SOOI, la capacité d'Ae. albopictus à transmettre efficacement ce virus a été démontrée (Pagès et al., 2006; Reiter et al., 2006; Vazeille et al., 2007; Grandadam \& Delatte, non publié).

Aedes albopictus (Skuse, 1894) est un moustique originaire des forêts d'Asie du Sud-Est (Smith, 1956) qui s'est progressivement adapté à l'environnement humain qui prodigue sources de sang (homme et animaux domestiques) et collections d'eau nécessaires au développement des larves. De nos jours, en Asie, Ae. albopictus est l'espèce la plus communément rencontrée dans des aires suburbaines et rurales (Tewari et al., 2004), mais aussi dans les villes où persiste une végétation arbustive comme à Kuala Lumpur, Singapour ou Tokyo. Ce rapprochement de l'homme lui a permis de coloniser de nouveaux territoires au gré des déplace- 
ments humains et de l'intensification des échanges commerciaux. Le meilleur exemple de ce phénomène est le transport intercontinental de pneus usagés qui constituent d'excellents sites de pontes dans lesquels les oufs peuvent subsister plusieurs semaines à l'état quiescent (Reiter, 1998). De nos jours, Aedes albopictus est présent dans de nombreuses îles du Pacifique (Hawaï, Salomon, Fidji), en Australie, sur le continent Américain depuis 1985 et en Afrique depuis 1989. L'Europe n'est pas épargnée puisque $A e$. albopictus est installé en Albanie depuis 1979, en Italie depuis 1990, en Espagne depuis 2004 ainsi qu'en France métropolitaine (Schaffner, 2005; Benedict et al., 2007). Cette récente expansion géographique de l'Asie vers les autres continents a suscité plusieurs études de phylogéographie visant à déterminer l'origine des différents épisodes de colonisation. Une étude basée sur des marqueurs isoenzymatiques suggère que les populations issues des États-Unis et du Brésil seraient originaires du Japon (Kambhampati et al., 1991). Une étude similaire, montrant que des populations issues de zones tempérées (Italie, Japon et États-Unis) formaient un groupe distinct de populations issues d'Asie tropicale (Indonésie), a conforté en partie ces premiers résultats (Urbanelli et al., 2000). Plus récemment, une étude basée sur l'emploi de marqueurs mitochondriaux remet en cause l'origine commune des populations présentes en Amérique du Nord et en Amérique du Sud (Birungi \& Munstermann, 2002). En utilisant des marqueurs similaires, les travaux de Mousson et al. (2005) suggèrent par ailleurs que les populations du Brésil sont en fait plus proches de populations originaires d'Asie du SudEst. L'existence d'une diapause hivernale des œufs issus des populations des États-Unis et l'absence de ce phénomène pour celles du Brésil suggèrent bien une origine tempérée pour les premières et une origine tropicale pour les secondes (Hawley, 1988). Concernant l'OI, il est admis que l'installation d'Ae. albopictus dans les îles de cette zone remonterait à plusieurs siècles tirant profit des épisodes migratoires de populations humaines en provenance d'Asie du Sud-Est (Edwards, 1920). Cependant, des spécimens de Madagascar et de la Réunion se sont avérés non différenciables génétiquement de spécimens originaires des États-Unis et de France (Mousson et al., 2005), soulignant les incertitudes liées à la question de l'origine géographique des populations d'Ae. albopictus actuellement présentes dans les îles du SOOI. Deux hypothèses peuvent être avancée : 1) la colonisation du SOOI par Ae. albopictus est contemporaine de la colonisation de ces îles par l'homme, il y a plusieurs siècles; 2) les populations actuelles présentes dans le SOOI sont issues d'au moins deux épisodes de colonisation, le premier remontant à plusieurs siècles, le second aux dernières décennies comme en Amérique et en Europe. La découverte en Afrique du Sud de spécimens dans des pneus en pro- venance du Japon et l'invasion récente de Mayotte illustrent bien les opportunités d'introduction dans la sous-région (Savage et al., 1992). À la Réunion, il a été démontré que les populations d'Ae. albopictus étaient fortement structurées notamment en fonction de facteurs environnementaux tels que la pluviométrie qui conditionne la disponibilité en eau et, par voie de conséquence, la dispersion des moustiques et les flux de gènes (Paupy et al., 2001).

L'implication d'Ae. albopictus dans la transmission du CHIKV est aujourd'hui confirmée par l'épisode épidémique qui a sévi à la Réunion. Parmi les 12 espèces de Culicidae présentes sur l'île, Ae. albopictus est l'espèce dominante alors qu'Ae. aegypti ne persiste que sous forme de populations résiduelles (Salvan \& Mouchet, 1994). Une étude visant à définir la répartition d'Ae. albopictus à La Réunion a montré, qu'en été, il était présent dans toute la zone anthropisée de l'île. En hiver, l'espèce reste active jusqu'à jusqu'à $1200 \mathrm{~m}$ malgré des températures moyennes pouvant atteindre $13^{\circ} \mathrm{C}$ (Delatte et al., 2007). Une enquête récente, établissant une typologie des gîtes larvaires, a montré la capacité d'Ae. albopictus à coloniser de nombreux types de collections d'eau, qu'elles soient d'origine anthropique ou naturelle, et ce dans des zones aussi bien fortement urbanisées que rurales. L'étude a par ailleurs montré une préférence pour les collections d'eau ombragées contenant une eau claire avec peu de matières organiques (Delatte et al., 2007). Dans les environnements urbains, les larves du moustique occupent préférentiellement des soucoupes sous les pots de fleurs et des creux de bambous et de rochers dans les environnements périurbains et ruraux (figure 2).

\section{TRANSMISSION VIRALE ET COMPÉTENCE VECTORIELLE}

Lors de l'épisode épidémique de dengue en 2004 à la Réunion, la présence du virus DEN-1 a été mise en évidence chez Ae. albopictus par le Centre national de référence des arbovirus de Lyon (Pierre et al., 2005), confirmant son rôle de vecteur sur l'île. Par ailleurs, les résultats de recherche du virus chikungunya à partir de lots de moustiques collectés sur le terrain durant la phase épidémique à la Réunion ont confirmé le rôle de cette même espèce dans la transmission du CHIKV. Ainsi, la recherche du virus par RT-PCR a permis de le mettre en évidence dans 24 lots constitués de 370 moustiques, sur un total de 117 lots d'adultes de l'espèce capturés en 2006 dans les foyers de transmission (1413 moustiques). De plus, la transmission verticale du virus, de la femelle à sa descendance, a été détectée en faible proportion, puisque sur 502 lots d'adultes issus de larves d'Ae. albopictus collectées sur 
$\mathbf{A}$

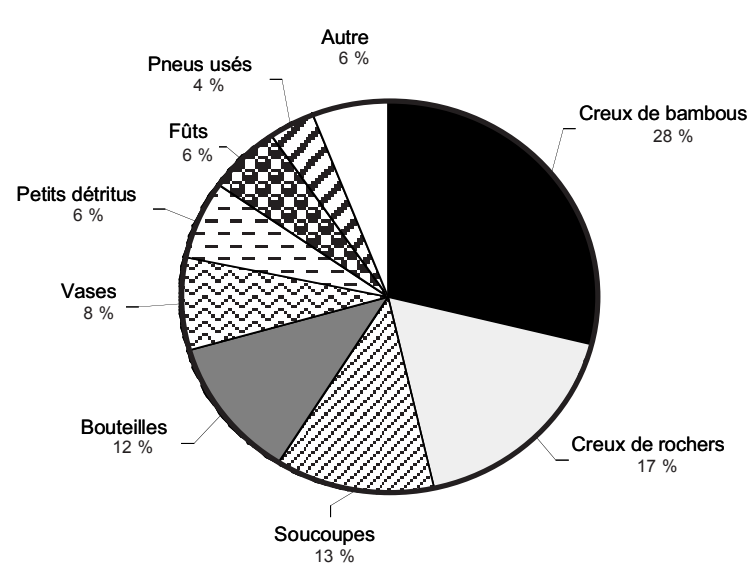

B

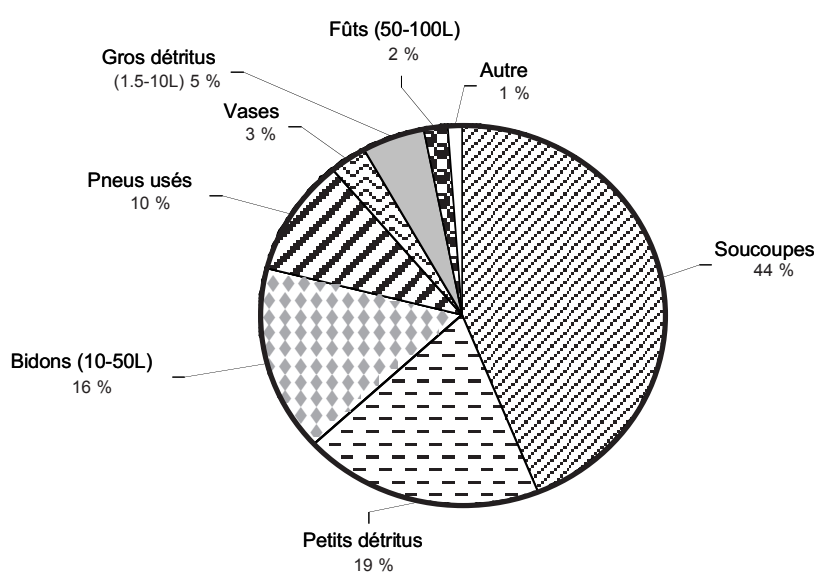

Fig. 2. - Typologie des gîtes larvaires d'Aedes albopictus à l'île de la Réunion en zone péri-urbaine/rurale (A) et en zone urbaine (B).

le terrain (6853 larves au total), deux contenaient du virus (Grandadam \& Delatte, non publié).

La compétence vectorielle, c'est-à-dire l'aptitude intrinsèque d'un arthropode hématophage à assurer le développement d'un virus et sa transmission est sous-tendue par des facteurs génétiques. Ainsi, chez Ae. aegypti, la compétence pour le virus de la dengue serait sous le contrôle de plusieurs gènes (Bennett et al., 2005). Chez cette même espèce, il a été démontré que la réceptivité au CHIKV est un trait dominant sous le contrôle d'un gène majeur situé sur le chromosome III (Mourya et al., 1994). Aedes albopictus est considéré comme un vecteur secondaire du CHIKV après $A e$. aegypti, notamment en Asie. Cependant, des études d'infections expérimentales montrent qu'Ae. albopictus est plus réceptif au CHIKV qu'Ae. aegypti (Tesh et al., 1976; Konishi \& Yamanishi, 1986; Turell et al., 1992) et ceci est d'autant plus prononcé vis-à-vis du génotype asiatique (Tesh et al., 1976). Des variations de compétence vectorielle pour le CHIKV sont observées en fonction de l'origine géographique des populations d'Ae. aegypti (Banerjee et al., 1988) et d'Ae. albopictus (Mangiafico, 1971; Tesh et al., 1976; Turell et al., 1992). Il existe également, pour une même souche de moustiques, des variations d'efficacité en fonction de l'origine des souches virales (Mourya, 1987). Des expériences récentes, réalisées à partir de populations d'Ae. albopictus de la Réunion, ont montré que la compétence vectorielle vis-à-vis du CHIKV variait en fonction de la souche virale et en fonction de la population de moustiques (Vazeille et al., 2007). Des moustiques récoltés pendant le pic épidémique (en mars 2006) se sont avérés extrêmement réceptifs vis-à-vis d'une souche virale isolée en novembre 2005. Cette souche, correspondant au génotype viral majoritaire lors de l'acmé de l'épidémie, présente un changement d'un acide aminé par rapport au génotype circulant en début d'épidémie en position 226 dans la glycoprotéine d'enveloppe E1 (Schuffenecker et al., 2006). Une réplication plus importante de ce génotype pourrait expliquer l'extrême efficacité de transmission des Ae. albopictus réunionnais.

Bien que la compétence vectorielle des populations d'Ae. albopictus originaire du SOOI pour le CHIKV n'avait jusqu'à présent jamais été évaluée, des résultats concernant la dengue étaient quant à eux disponibles. Des études ont démontré que des populations vectorielles originaires de la Réunion (Paupy et al., 2001) et de Madagascar (Vazeille et al., 2001) présentaient une réceptivité orale vis-à-vis de la dengue supérieure à celle des populations en provenance d'Asie du Sud-Est (Vazeille et al., 2003). Tout comme pour le CHIKV, des variations de compétence vectorielle sont observées pour les DENV en fonction de l'origine géographique d'Ae. aegypti et d'Ae. albopictus (Paupy et al., 2001; Failloux et al., 2002). Néanmoins, au laboratoire, $A e$. aegypti semble être globalement un meilleur vecteur des DENV qu'Ae. albopictus (Chen et al., 1993). Bien que la compétence vectorielle constitue un élément clé, d'autres facteurs relatifs à l'écologie (distribution, dynamique des populations,...), à la biologie (comportement trophique, cycle trophogonique, longévité, dispersion,...) et à l'environnement du vecteur se surajoutent pour déterminer l'efficacité de la transmission en conditions naturelles, c'est-à-dire la capacité vectorielle.

La biologie d'Ae. albopictus a fait l'objet de nombreuses observations sur le terrain et au laboratoire dans le monde (Hawley, 1988). La biologie et l'écologie des populations réunionnaises est en cours d'étude. Les premiers résultats, en adéquation avec les précédentes études, mettent en avant la très grande plasticité écologique ainsi que l'importante capacité d'adaptation de ce moustique. Il est par conséquent hasardeux de géné- 
raliser des données de la biologie d'Ae. albopictus obtenues localement à l'ensemble des populations présentes dans l'aire de répartition géographique de l'espèce. La compréhension des systèmes vectoriels $A e$. albopictus-CHIKV et Ae. albopictus-DENV, ainsi que la mise en place d'outils de surveillance et de lutte antivectorielle doivent tenir compte de la notion d'adaptation locale, c'est pourquoi des collaborations étroites ont été mises en place entre les organismes de recherche et de lutte anti-vectorielle.

\section{LA LUTTE ANTI-VECTORIELLE À LA RÉUNION}

D epuis l'éradication du paludisme à la Réunion, qui a été établie par l'Organisation mondiale de la santé (OMS) en 1979, le service de lutte antivectorielle (LAV) de la Direction régionale des affaires sanitaires et sociales (DRASS) de la Réunion avait pour principal objectif la lutte contre la réintroduction du paludisme, dans la mesure où cette maladie est endémique dans de nombreux pays de l'OI et où le principal vecteur, Anopheles arabiensis, se maintient en milieu rural. Les épidémies de dengue (2004) et de chikungunya (2005-2007) ont souligné la nécessité d'étendre les actions d'éducation sanitaire, de surveillance et de démoustication aux autres vecteurs présents sur l'île, notamment Ae. albopictus en milieu urbain (figure 3). À la Réunion, l'omniprésence d'Ae. albopictus dans toutes les zones urbaines jusqu'à 1200 m d'altitude, et

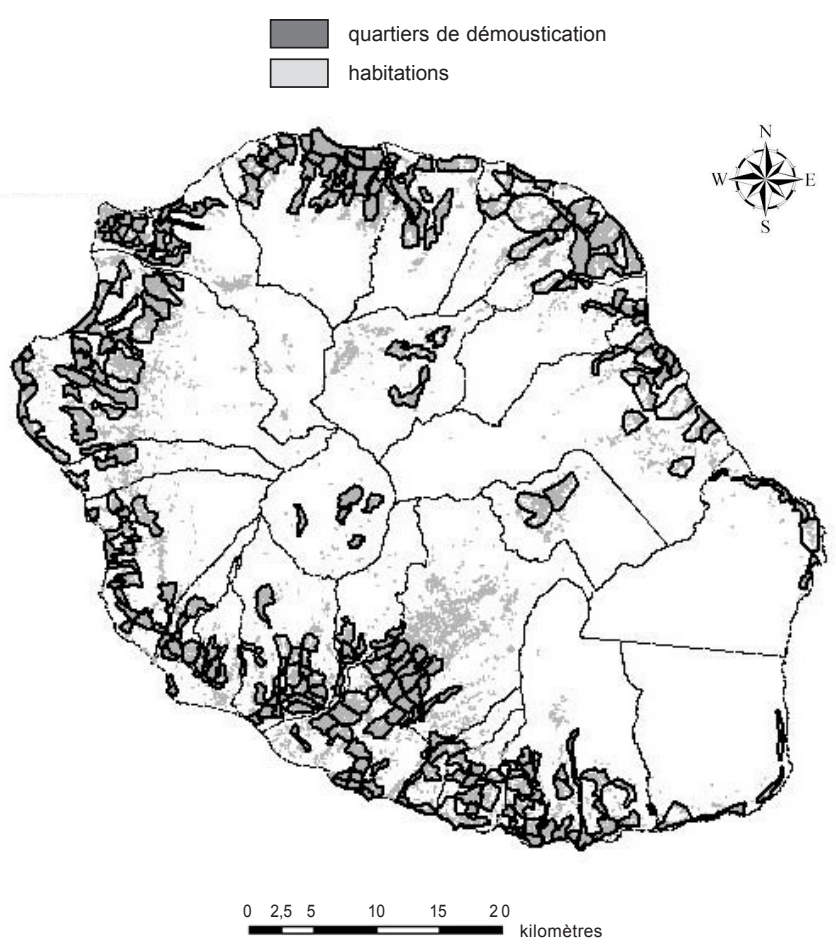

Fig. 3. - Localisation géographique des quartiers soumis à une démoustication. durant toute l'année sur le littoral, oblige les services en charge de la LAV à focaliser la lutte contre ce vecteur dans les zones urbaines où l'espèce colonise essentiellement des gîtes larvaires anthropiques (Rodhain, 2001).

\section{LA SURVEILLANCE ENTOMOLOGIQUE}

I 'épidémie de dengue de 2004 a suscité la création d'un réseau de surveillance entomologique spécifique du vecteur Ae. albopictus. Ce réseau est fondé sur la mesure mensuelle d'indices d'infestation larvaires dans 16 quartiers, chacun subdivisé en souszones (figure 4). Initiée en décembre 2004, la surveillance a dû être suspendue lors de la survenue de l'épidémie de chikungunya en mai 2005 , au profit d'actions de lutte péri-focale. En août 2006, dès la fin de l'épidémie de chikungunya, la surveillance entomologique était réactivée et étendue selon un réseau de 42 quartiers (225 zones) répartis sur toute l'île (figure 5). La sélection des quartiers s'est faite en fonction de critères relatifs à la typologie de l'habitat, à l'environnement et à l'intensité de la transmission de la dengue et du chikungunya. Des suivis y sont mensuellement établis afin d'évaluer l'évolution spatio-temporelle des densités vectorielles et de la nature des gîtes larvaires dans le but d'optimiser les actions de lutte et de sensibilisation de la population. Ainsi, chaque mois, 150 à 200 évaluations (mesures des indices larvaires sur une soixantaine de maisons) sont réalisées. Dans l'avenir, la surveillance devrait être focalisée sur une quinzaine de quartiers

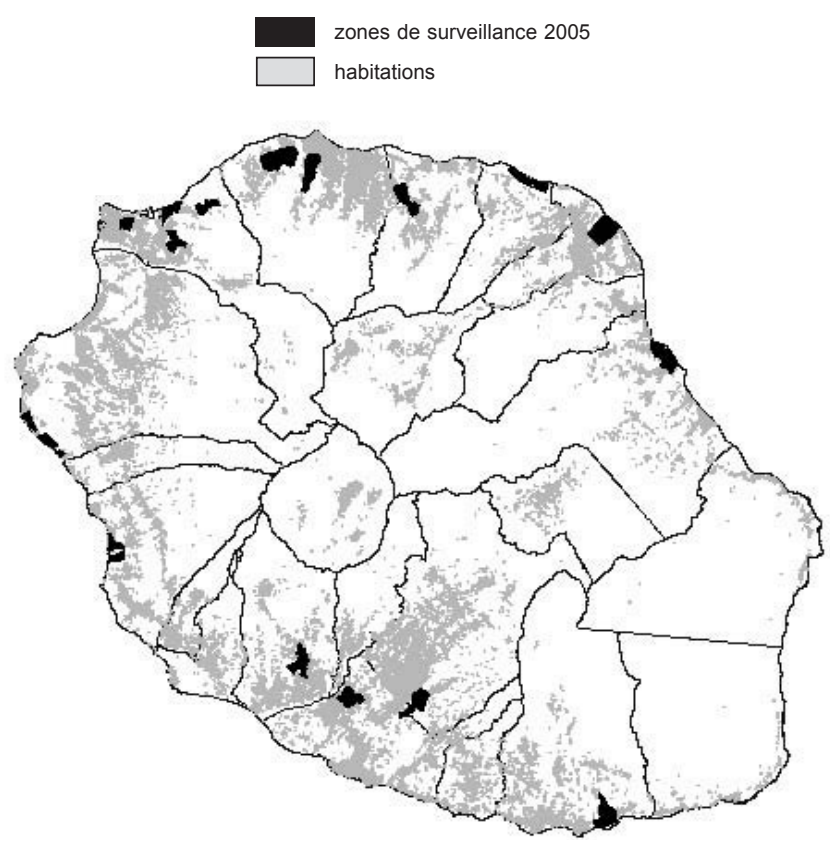

Fig. 4. - Réseau d'entomo-surveillance de 2005-2006. 


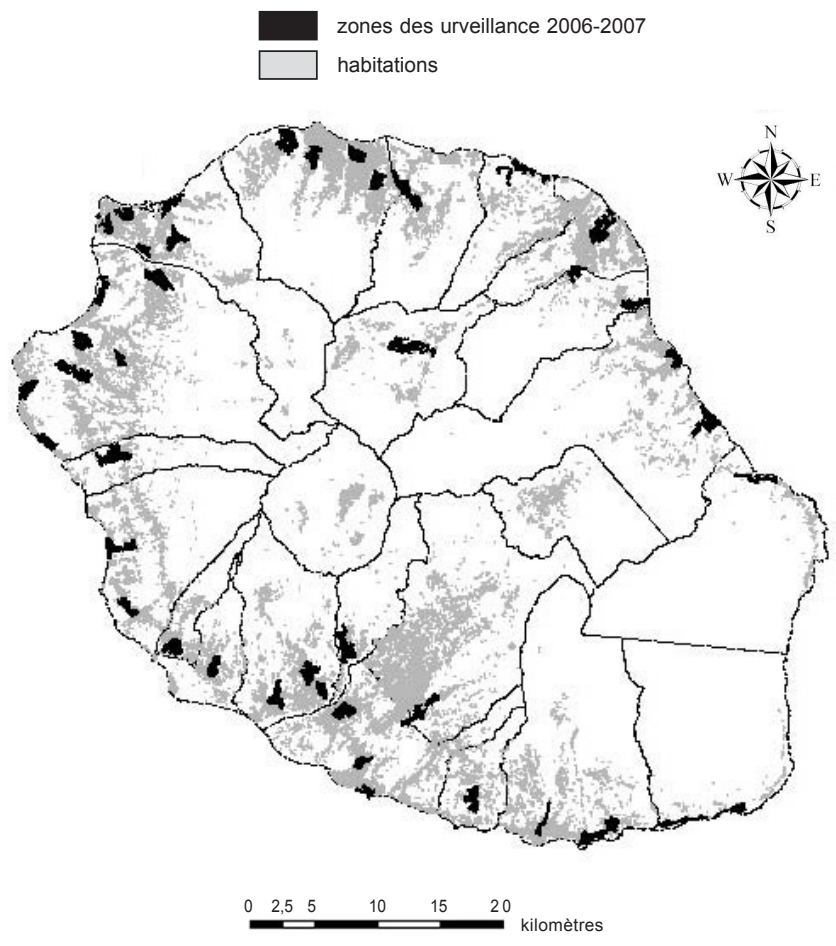

Fig. 5. - Réseau d'entomo-surveillance de 2006-2007.

représentatifs du milieu urbain réunionnais incluant les gites naturels en milieu urbain (ravines), et le calcul d'infestation larvaire devrait être amélioré en tenant compte de la productivité nymphale des gîtes à l'aide d'indices pondérés (Yébakima, 1996).

\section{LES PROTOCOLES DE LUTTE}

I nitiée en 2004, la lutte contre Ae. albopictus s'est continuellement adaptée aux contextes épidémiologiques en adoptant de nouvelles méthodes et techniques. Quatre évolutions majeures sont à relever depuis l'épidémie de DENV (2004) jusqu'à la fin de l'épidémie de CHIKV en 2007 (tableau I).

De 2004 à février 2006, les mesures consistaient essentiellement au traitement péri-focal à l'aide d'adulticides et de larvicides autour de chaque cas déclaré et dans les jardins des dix maisons voisines. Les collectivités locales ont été mises à contribution pour augmenter les rythmes de collecte des déchets et nettoyer les gîtes productifs recensés, tels que carcasses de véhicules, dépôts sauvages, etc.

De février 2006 à mai 2006, le traitement péri-focal ne permettant plus de répondre efficacement à l'élévation brutale du nombre de cas (47000 cas pour la $5^{\text {ème }}$ semaine de 2006), les moyens humains et matériels de la LAV ont été renforcés jusqu'à 3000 hommes au plus fort de l'épidémie (incluant les forces armées (FAZSOI)), pour une intervention généralisée sur l'ensemble des
1066 zones urbaines de La Réunion. Un numéro d'appel gratuit permettait d'obtenir des informations, de solliciter une intervention et de signaler les cas. Une campagne massive d'information à été réalisée.

De juin 2006 à novembre 2006, la diminution du nombre de cas pendant l'hiver austral (100 cas par semaine) et la réduction progressive des moyens mobilisés ont concouru à la mise en place du protocole dit de "vigilance renforcée", avec un retour à des interventions ciblées autour des cas, et à des actions préventives dans les zones où la surveillance entomologique détectait des densités larvaires élevées. Des traitements antilarvaires ont été mis en place dans plus de 200 ravines situées en milieu urbain, et l'élimination des gites péridomestiques s'est poursuivie. Des campagnes d'information selon la méthode COMBI (communication for behavioural impact) ont été réalisées et modulées en fonction de la situation épidémique, afin d'impliquer la société réunionnaise dans les actions de démoustication.

De décembre 2006 à juillet 2007, la baisse du nombre de cas (de moins de 30 à 0 cas hebdomadaire) et la création d'un Groupement d'intérêt public (GIP) coordonnant toutes les mesures de LAV dans le département signent un retour aux missions "socles" de la lutte contre les vecteurs : traitements préventifs anti-larvaires étendus, interventions ciblées en fonction des indices entomologiques et des cas, incitation forte à la lutte communautaire par la mise en place d'opérations de démoustication associant la population, les collectivités et le milieu associatif. Les traitements adulticides ont été limités et orientés en fonction de critères entomologiques et épidémiologiques (autour des cas signalés) (tableau I). Le programme de contrôle des gîtes très productifs en moustiques a été renforcé (297 ravines et canaux suivis chaque semaine). Des opérations spéciales de traitement et de sensibilisation ont été réalisées autour des sites touristiques, dans les cimetières où existent de nombreux gîtes larvaires créés par les vases et dans les quartiers à forte production de moustiques. La mise en ouvre de cette stratégie a cependant rencontré des contraintes liées à la plasticité écologique du vecteur, à sa forte adaptabilité au milieu urbain, et enfin à la difficulté d'accéder aux jardins privatifs ne permettant pas le traitement exhaustif des zones.

\section{CONCLUSION}

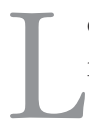

es données actuelles montrent clairement la vulnérabilité de la Réunion et des îles de l'Océan Indien vis-à-vis d'épidémies d'arboviroses transmises par les Aedes. Néanmoins, la prédiction d'épidémies de l'ampleur de celles enregistrées en 1977-1978 pour la dengue ou de celle de 2005-2006 pour le chi- 


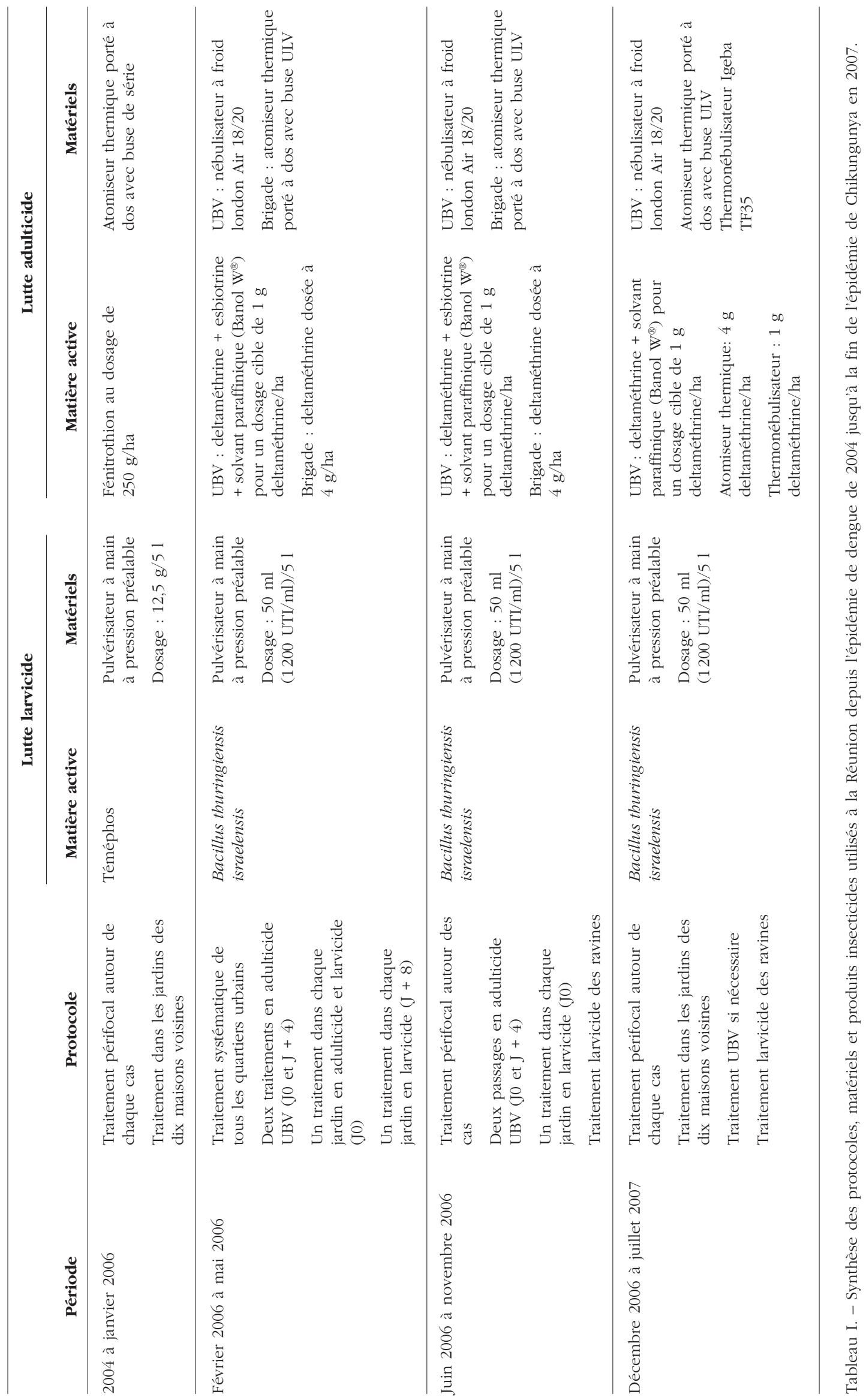


kungunya est actuellement difficilement envisageable dans l'Océan Indien. L'épidémie de chikungunya a révélé la nécessité de développer des recherches approfondies sur les vecteurs, de mener au niveau local et régional une réflexion sur la gestion de l'environnement et des déchets, de pérenniser la lutte contre les moustiques et de maintenir une veille sanitaire régionale afin de pouvoir au mieux prévenir une épidémie de cette ampleur. Un plan de prévention des arboviroses est en cours de réalisation. Il devra permettre d'intervenir plus rapidement dès la réception des premiers signaux d'alerte, et d'adapter la réponse en fonction du virus infectieux et de son vecteur.

\section{REMERCIEMENTS}

U ne grande partie de ce travail a été réalisée dans le cadre du projet Entomochik, financé par l'Agence nationale de la recherche (ANR), programme SEST.

\section{RÉFÉRENCES}

Arankalle V.A., Shrivastava S., Cherian S., Gunjikar R.S., Walimbe A.M., Jadhav S.M., Sudeep A.B. \& Mishra A. Genetic divergence of Chikungunya viruses in India (19632006) with special reference to the 2005-2006 explosive epidemic. Journal of General Virology, 2007, 88, 1967-1976.

Banerjee K., Mourja D.T. \& MalunjKar A.S. Susceptibility and transmissibility of different geographical strains of Aedes aegypti mosquitoes to Chikungunya virus. Indian Journal of Medical Research, 1988, 87, 134-138.

Benedict M., Levine R.S., Hawley W.A. \& Lounibos L.P. Spread of the Tiger: global risk of invasion by the mosquito Aedes albopictus. Vector Borne and Zoonotic Diseases, 2007, 7, 76-85.

Bennett K.E., Flick D., Fleming K.H., Jochim R., Beaty B.J. \& BlaCK W.C. Quantitative trait loci that control dengue-2 virus dissemination in the mosquito Aedes aegypti. Genetics, 2005, 170, 185-194.

Birungi J. \& Munstermann L.E. Genetic structure of Aedes albopictus (Diptera: Culicidae) populations based on mitochondrial ND5 sequences: evidence for an independent invasion into Brazil and United States. Annals of the Entomological Society of America, 2002, 95, 125-132.

Boisier P., Morvan J., Laventure S., Charrier N., Martin E., Ouledi A. \& Roux J. Dengue 1 epidemic in the Grand Comoro Island (Federal Islamic Republic of the Comores). Annales de la Société Belge de Médecine Tropicale, 1994, 74, 217-229.

Calisher C.H., Niuti M., Lazuick J.S., Ferrari J.D.M. \& Kappus K.D. Dengue in the Seychelles. Bulletin of the World Health Organization, 1981, 59, 619-622.

Chen W.J., Wei H.L., Hsu E.L. \& Chen E.R. Vector competence of Aedes albopictus and Ae. aegypti (Diptera: Culi- cidae) to dengue 1 virus on Taiwan: development of the virus in orally and parenterally infected mosquitoes. Journal of Medical Entomology, 1993, 30, 524-530.

Cotholendy. Relation de l'épidémie de dengue qui a régné à Saint-Denis (Réunion) pendant les mois de février, mars, avril et mai 1873. Archives de Médecine Navale, 1873, 20, 190.

Coulanges P., Clerc Y., Jousset F.X. \& Rodhain F. Dengue à la Réunion. Isolement d'une souche à l'Institut Pasteur de Madagascar. Bulletin de la Société de Pathologie Exotique, 1979, 72, 205-209.

Delatte H., Dehecg J.S., Thiria J., Domerg C., Paupy C. \& FonTENILLE D. Geographic distribution and developmental sites of Aedes albopictus (Diptera: Culicidae) during a Chikungunya epidemic event. Vector Borne and Zoonotic Diseases, 2007, 7, 1-11.

Diallo M., Thonnon J., Traore-Lamizana M. \& Fontenille D. Vectors of Chikungunya virus in Senegal: current data and transmission cycles. American Journal of Tropical Medicine and Hygiene, 1999, 60, 281-286.

EDWARDS F.W. Notes on the mosquitoes of Madagascar, Mauritius and Reunion. Bulletin of Entomological Research, 1920, 11, 133-138.

Failloux A.B., Vazeille M. \& Rodhain F. Geographic genetic variation in populations of the dengue virus vector Aedes aegypti. Journal of Molecular Evolution, 2002, 55, 653-663.

Fontenille D., Failloux A.B. \& Romi R. Should we expect Chikungunya and Dengue in Southern Europe? In : Emerging Pests and Vector-Borne Diseases in Europe. Takken W. \& Knols B.G.J. (eds), Wageningen Academic Publishers, Wageningen, The Netherlands, 2007, 169-184.

GibBons R.V. \& VAughn D.W. Dengue: an escalating problem. British Medical Journal, 2002, 324, 1563-1566.

Gubler D. Dengue and dengue hemorrhagic fever. Clinical Microbiology Reviews, 1998, 11, 480-496.

Halstead S.B., Scanlon J.E., Umpaivit P. \& Udomsakdi S. Dengue and Chikungunya virus infection in man in Thailand, 1962-1964. IV. Epidemiologic studies in the Bangkok metropolitan area. American Journal of Tropical Medicine and Hygiene, 1969, 18, 997-1021.

Hammon W.M., Rudnick A. \& SAther G. New hemorrhagic fevers of children in the Philippines and Thailand. Transaction of the Association of American Physicians, 1960, 73, 140-155.

HAWLEY A.H. The biology of Aedes albopictus. Journal of the American Mosquito Control Association, 1988, 4, 2-39.

Holmes E.C. \& TwIDDY S.S. The origin, emergence and evolutionary genetics of dengue virus. Infection, Genetics and Evolution, 2003, 3, 19-28.

HotTa S. \& Kimura R. Experimental studies on dengue-1. Isolation identification and modification of the virus. Journal of Infectious Diseases, 1952, 90, 1-9.

Johnston R.E. \& Peters C.J. Alphaviruses associated primarily with fever and polyarthritis, in: Fields B.N., Knipe D.M., Howley P.M. (eds), Fields Virology, Lippincott Williams \& Wilkins, Philadelphia, 1996.

Julvez J., Ragavoodoo C., Gopaul A.R. \& Mouchet J. Maladies humaines transmises par les culicidés dans les îles du sud-ouest de l'Océan Indien. Bulletin de la Société de Pathologie Exotique, 1998, 91, 88-103. 
Jupp P.G. \& McIntosh B.M. Chikungunya disease, in: The Arboviruses: epidemiology and ecology. MTP (ed.), CRC Press, Boca Raton, Florida, 1988, 137-157.

Kalunda M., Lwangassozi C., Lule M. \& Mukuye A. Isolation of Chikungunya and Pongola viruses from patients in Uganda. Transactions of the Royal Society of Tropical Medicine and Hygiene, 1985, 79, 567-567.

KambHampati S., Black W.C. \& Rai K.S. Geographic origin of the United-States and Brazilian Aedes albopictus inferred from allozyme analysis. Heredity, 1991, 67, 85-94.

Konishi E. \& Yamanishi H. Titer distribution analysis of Chikungunya virus in Aedes albopictus (Diptera: Culicidae). Journal of Medical Entomology, 1986, 23, 92-98.

Laras K., Sukri N.C., Larasati R.P., Bangs M.J., Kosim R., Djauzi S., Wandra T., Master J., Kosasih H., Hartati S., BecKetT C., Sedyaningsih E.R., Beecham H.J. \& Corwin A.L. Tracking the re-emergence of epidemic Chikungunya virus in Indonesia. Transactions of the Royal Society of Tropical Medicine and Hygiene, 2005, 99, 128-141.

LEROY E. 2007. Chikungunya - Africa (Gabon): (http://www.promedmail.org). Article ProMed 20070519.1591, du 19 mai 2007.

MACCARTHY D.D. \& BRENT R.H. An account of an outbreak of dengue fever in Dzaoudzi, Comoro Islands, January 1943. East African Medical Journal, 1946, 20, 293-298.

MangIAfICO J.A. Chikungunya virus infection and transmission in five species of mosquitoes. American Journal of Tropical Medicine and Hygiene, 1971, 20, 642-645.

Metselaar D., Grainger C.R., Oei K.G., Reynolds D.G., Pudney M., Leake C.J., Tukei P.M., D'offray R.M. \& Simpson D.I.H. An outbreak of type 2 Dengue fever in the Seychelles, probably transmitted by Aedes albopictus (Skuse). Bulletin of the World Health Organization, 1980, 58, 937-943.

Monath T.P. Dengue: the risk to developed and developing countries. Proceedings of the National Academic of Sciences USA, 1994, 91, 2395-2400.

Moore D.L., Reddy S., Akinkugb F.M., Lee V.H., Davidwes T.S., CAusey O.R. \& CAREY D.E. Epidemic of Chikungunya fever at Ibadan, Nigeria, 1969. Annals of Tropical Medecine and Parasitology, 1974, 68, 59-68.

Mora B. [L'épidémie de dengue à l'île de La Réunion], Université de Bordeaux II, Bordeaux, 1979, pp. 60.

Mourya D.T. Absence of transovarial transmission of Chikungunya virus in Aedes aegypti and Ae. albopictus mosquitoes. Indian Journal of Medical Research, 1987, 85, 593-595.

Mourya D.T., Gokhale M.D., Malunjkar A.S., Bhat H.R. \& BANERJEE K. Inheritance of oral susceptibility of Aedes albopictus to Chikungunya virus. American Journal of Tropical Medicine and Hygiene, 1994, 5, 295-300.

Mousson L., Dauga C., Garrigues T., Schaffner F., Vazeille M. \& Failloux A.B. Phylogeography of Aedes (Stegomyia) aegypti (L.) and Aedes (Stegomyia) albopictus (Skuse) (Diptera: Culicidae) based on mitochondrial DNA variations. Genetical Research, 2005, 86, 1-11.

Pages F., Corbel V. \& Paupy C. Aedes albopictus : chroniques d'un vecteur expansionniste. Médecine Tropicale, 2006, 66, 226-228.

Pastorino B., Muyembe-Tamfum J.J., Bessaud M., Tock F., Tolou H., Durand J.P. \& Peyrefitte C.N. Epidemic resur- gence of Chikungunya virus in Democratic Republic of the Congo: Identification of a new central African strain. Journal of Medical Virology, 2004, 74, 277-282.

Paupy C., Girod R., Salvan M., Rodhain F. \& Failloux A.B. Population structure of Aedes albopictus from Reunion Island (Indian Ocean) with respect to susceptibility to a Dengue virus. Heredity, 2001, 87, 273-283.

Perrau J., Catteau C., Michault A., Parain A. \& Favier F. Fin 2006, 300000 personnes avaient été atteintes par le chikungunya. Économie de la Réunion, 2006, 129.

Peyrefitte C.N., Rousset D., Pastorino B., Poulllot R., Bessaud M., Tock F., Mansaray H., Merle O.L., Pascual A.M., Paupy C., Vessiere A., Imbert P., Tchendjou P., Durand J.P., Tolou H. \& Grandadam M. Chikungunya virus, Cameroon, 2006. Emerging Infectious Diseases, 2006, 13, 768-771.

Pialoux G., Gauzere B.A. \& Strobel M. Chikungunya virus infection: review through an epidemic. Médecine et Maladies Infectieuses, 2006, 36, 253-263.

Pierre V., Thiria J., Rachou E., Sissoko D., Lassalle C. \& Renault P. Épidémie de dengue-1 à la Réunion en 2004, Journées INVS, 2005. http://www.invs.sante.fr/publications/2005/jvs_2005/poster_13.pdf

Pougnet V. Note de l'épidémie de dengue qui a régné à PortLouis en 1872-1873. Bulletin de la Société Médicale de l'île Maurice, 1890, 8, 448-450.

RAO T.R. Recent epidemics caused by Chikungunya virus in India 1963-1965. Scientific Culture, 1966, 32, 215.

ReITER P. Aedes albopictus and the world trade in used tires, 1988-1995: the shape of things to come? Journal of the American Mosquito Control Association, 1998, 14, 8394.

ReIter P., Fontenille D. \& Paupy C. Aedes albopictus as an epidemic vector of Chikungunya virus: another emerging problem? Lancet Infectious Disease, 2006, 6, 463-464.

Robinson C.M. An epidemic of virus disease in Southern Province, Tanganyika Territory in 1952-53. I. Clinical features. Transactions of the Royal Society of Tropical Medicine and Hygiene, 1955, 49, 28-32.

RoDHAIN F. Fièvre jaune, dengue et autres arboviroses exotiques. Encyclopédie Médico-Chirurgicale, 2001.

Ross R.W. The Newala epidemic. III. The virus isolation, pathogenic properties and relationship to the epidemic. Journal of Hygiene, 1956, 54, 177-191.

SABIN A.B. Research on dengue during World War II. American Journal of Tropical Medicine and Hygiene, 1952, 1, 30-50.

Salvan M. \& Mouchet J. Aedes albopictus et Aedes aegypti à l'île de la Réunion. Annales de la Société Belge de Médecine Tropicale, 1994, 74, 323-326.

Savage H.M., Ezike V.I., Nwankwo A.C., Spiegel R. \& Miller B.R. First record of breeding populations of Aedes albopictus in continental Africa: implications for arboviral transmission. Journal of the American Mosquito Control Association, 1992, 8, 101-103.

SCHAFFNER F. Rapport technique sur la présence du moustique Aedes albopictus dans les Alpes-Maritimes, bilan au 28 septembre, 2005, 1-3. 
Schuffenecker I., Iteman I., Michault A., Murri S., Frangeul L., Vaney M.-C., Lavenir R., Pardigon N., Reynes J.-M., Pettinelli F., Biscornet L., Diancourt L., Michel S., Duquerroy S., Guigon G., Frenkiel M.-P., Brehin A.-C., Cubito N., Despres P., Kunst F., Rey F.l.A., Zeller H. \& Brisse S. Genome microevolution of Chikungunya viruses causing the Indian Ocean outbreak. PLoS Medicine, 2006, 3, 1-13.

Sergon K., Yahaya A., Brown J., Bedja S., Mlindasse M., Agata N., Allaranger Y., Ball M., Powers A., Ofula V., Onyango C., Konongoi L., SAng R., NJenga M. \& Breiman R. Seroprevalence of Chikungunya virus infection on Grande Comore Island, union of the Comoros, 2005. American Journal of Tropical Medicine and Hygiene, 2007, 76, 1189-1193.

Skuse F. The banded mosquito of Bengal. Indian Museum Notes, 1894, 3, 20.

SMith C.E.G. The history of dengue in tropical Asia and its probable relationship to the mosquito Aedes aegypti. Journal of Tropical Medecine and Hygiene, 1956, 59, 243251.

Tesh R.B., Gubler D.J. \& Rosen L. Variation of geographic strains of Aedes albopictus in susceptibility in infection with chikungunya virus. American Journal of Tropical Medicine and Hygiene, 1976, 25, 326-335.

Tewari S.C., Thenmozhi V., Kathoi C.R., Manavalan R., Munirathinam A. \& Gajanana A. Dengue vector prevalence and virus infection in a rural area in South India. Tropical Medecine and International Health, 2004, 9, 499-507.

Thonnon J., Spiegel A., Diallo M., Diallo A. \& Fontenille D. Épidémies à virus chikungunya en 1996 et 1997 au Sénégal. Bulletin de la Société de Pathologie Exotique, 1999, 92, 79-82.

Turell M.J., Beaman J.R. \& Tammariello R.F. Susceptibility of selected strains of Aedes aegypti and Aedes albopictus (Diptera: Culicidae) to Chikungunya virus. Journal of Medical Entomology, 1992, 29, 49-53.

Urbanelli S., Bellini R., Carrieri M., Sallicandro P. \& Celli G. Population structure of Aedes albopictus (Skuse): the mosquito which is colonizing Mediterranean countries. Heredity, 2000, 84, 331-337.

Vazeille M., Rosen L., Mousson L. \& Failloux A.B. Low oral receptivity for dengue type 2 viruses of Aedes albopictus from Southeast Asia compared with that of Aedes aegypti. American Journal of Tropical Medicine and Hygiene, 2003, 68, 203-208.

Vazeille M., Mousson L., Rakatoarivony I., Villeret R., RoDhain F., Duchemin J.B. \& Failloux A.B. Population genetic structure and competence as a vector for Dengue type-2 virus of Aedes aegypti and Aedes albopictus from Madagascar. American Journal of Tropical Medicine and Hygiene, 2001, 65, 491-497.

Vazeille M., Moutailler S., Coudrier D., Rousseaux C., Khun H., Huerre M., Thiria J., DehecQ J.S., Fontenille D., SchuffeNeCKer I., Despres P. \& Failloux A.B. Two Chikungunya isolates from the outbreak of la Reunion (Indian Ocean) exhibit different patterns of infection in the mosquito, Aedes albopictus? PLOS ONE, 2007, 2, e1168.

YéBAKIma A. Lutte contre Aedes aegypti en Martinique : apport des études entomologiques. Bulletin de la Société de Pathologie Exotique, 1996, 89, 161-162.
Yersin P.C., Bovet P., Herminie P. \& Zeller H. Public health importance of mosquito-borne diseases in the Seychelles (Indian Ocean). The Infectious Disease Review, 1999, 1, 189-199.

Zeller H.G. Dengue, arbovirus et migrations dans l'Océan Indien. Bulletin de la Société de Pathologie Exotique, 1998, 91, 56-60.

Reçu le 4 octobre 2007 Accepté le 7 novembre 2007 